\title{
Searching for Salient Aspects of Resonant Interaction
}

\author{
Caroline Hummels
}

Received: 15 December 2006 / Accepted: 15 March 2007 / Published online: 1 August 2007

(C) Springer Science + Business Media B.V. 2007

\begin{abstract}
This paper illustrates my quest for diversity, more specifically resonant interaction with products. Resonance occurs due to the perfect interplay between a person and a product. It evokes strong positive emotions, unique accompanying behaviour, heightened awareness and cognitive processing. In this paper, I first explain the importance of diversifying designs, especially now we are pursuing the next generation of digital products. I show that designing for resonance can be a way to achieve diversity. I clarify the concept of resonance and resonant interaction. Subsequently, I explain the importance of research through design to study this phenomenon, i.e., by designing in a way that results in experiential prototypes, and subsequently testing these prototypes, scientific knowledge can be generated. Through these prototypes, I study the relationship between the individual user with his concerns, values, personality, skills, senses and effectivities on the one hand, and the interaction and product characteristics on the other
\end{abstract}

C. Hummels $(\bowtie)$

Department of Industrial Design,

Technische Universiteit Eindhoven,

Den Dolech 2, HG 2.59,

5600 MB Eindhoven, The Netherlands

e-mail: C.C.M.Hummels@tue.nl

C. Hummels

Faculty of Industrial Design Engineering,

Delft University of Technology,

Delft, The Netherlands hand. The paper concludes with two studies illustrating the way of working and the kind of answers I am obtaining with respect to resonant interaction. It appears that one can design for personality and prolong positive emotions even after the interaction has stopped, which strengthens the case for searching for salient aspects of resonant interaction.

Keywords Resonant interaction · Products .

Diversifying design

\section{Design for Diversity}

I was deeply shocked when I realised that I had lost my sunglasses. I just could not believe that they were gone. How could they do that to me? But since August this year, when my family gave me almost the same sunglasses for my birthday, the world has become beautiful again. Although the name is atrocious, model 8569 with v6071 lenses, the design from Silhouette is absolutely magnificent. The frame is made of pure Flexon Titanium, which is extremely light. I hardly notice the 8 grams when wearing them, although I am aware of them every once in a while and enjoy the splendid feeling. Despite their weight, they are strong and not very vulnerable. Because they don't have hinges, they tend to unfold, and 
consequently have a perfect way of displaying themselves when lying on a table. Due to their construction, they come in a special hard case. When opening the case, the sunglasses curiously peek over the edge at you as if they want to explore the world. And every time I take them out of the case and gently put them on using both hands I experience a thrill, which is a perfect subtle feeling. I just feel more intense and present, and I have the impression that I become taller when wearing them. Because it was a bit troublesome to take the original glasses off and put them in the case every time the lighting conditions changed, for example when I entered or left a shop, I was extremely happy to discover that the new version had special polycarbonate polarized lenses that offer $100 \%$ UV protection. This not only protects me from glaring sunlight and accompanying headaches, but also enables me to see perfectly indoors. And even when I'm not wearing my sunglasses, and am carrying them around in my new Hester van Eeghen bag, I still get this warm feeling when thinking of them. I know that not everyone resonates with these glasses, they only received a rating of 3 on a 5 point scale on a sunglasses comparison website, but for me they are heaven.

I have, over the years, collected several products like my sunglasses, which have surrounded me, and with which I have a special bond. These products surprise me and even move me. They are able to distract me from the drudgery of daily life and show me its beauty. They resonate with me and it feels good.

Unfortunately, I cannot seem to get this special bond with most of the digital products I own. They remain rather distant and anonymous boxes. And what is worse, they often frustrate me. I have trouble operating these machines, and it apparently does not suffice to be an industrial design engineer to comprehend and control them. Why are they so complicated and why do they make me feel as if I have to conquer them instead of doing my job, let alone experiencing the thrill and pleasure of operating them? Why is it so difficult to find digital products that resonate with me?

Although technological development offers so many possibilities for designers, the latter do not seem to take advantage of them. Electronic circuits and microprocessors liberate the designer from the restrictions of a specific appearance of a product or a specific interaction with it. However, in practice, the diversity of digital products is minimal. The kind of interaction is based mainly on cognition using text-based interfaces. Why do these products not capitalize on the full range of human skills and abilities (Dourish 2001; Overbeeke et al. 2003)? Why aren't they personal pathways that allow individuals to find and create their own experiences (Hummels et al. 2003)? The scope offered to us by digital technology seems to have been barely explored for human product interaction.

I believe that diversity is of paramount importance when pursuing the next generation of digital products, especially when we are moving towards intelligent and adaptive products and smart surroundings. Although we are all humans (and thus have common sensorial and physical aspects) and live in the same world, we have our own personal history, our own concerns and values, and our own physical, emotional and rational characteristics. We are all different, so why are most digital products alike, particularly at the interaction level? In particular, products that adapt to the user and even anticipate their needs, require designers to gear the products to individuals, since 'the user' does not exist, thus resulting in diversity.

Designing for diversity demands extensive knowledge about the relationship between the individual user with his concerns, values, personality, skills, senses, effectivities on the one hand, and the interaction and product characteristics on the other. Within this design quest for diversity, I focus on resonant interaction. Before I elaborate on this kind of interaction and show why and how designers can design for resonance, let me first explain the concept of resonance.

\section{Resonance}

Resonance is a commonly known concept with different denotations and connotations, depending on the field of application (e.g. physics, acoustics, chemistry, astronomy and perception). Two of these fields spurred me on to study resonant interaction within a design context, i.e., physics and perception.

Within physics, resonance is a vibration of large amplitude in a mechanical or electrical system caused by a relatively small periodic stimulus of the same or nearly the same period as the natural vibration period 
of the system, according to Merriam-Webster Online Dictionary. A stimulus and a system that are in sync can have major consequences, as the first Tacoma Narrows suspension bridge demonstrated in 1940. This bridge near the city of Tacoma, Washington, which had only been open for traffic for a few months, collapsed due to wind-induced vibrations (Smith 1974).

Thus resonance occurs if you vibrate a system at just the right frequency: near one of its natural frequencies. Moreover, if an input is timed just right to reinforce the motion of a product, the motion builds up; a series of small inputs can create a large motion. We have all experienced the beauty of this last rule when trying to swing as high as possible when we were children. As a designer, I always love and aim to achieve the embodiment of this metaphor with respect to beautiful interaction: the perfect synchronisation between a person and a product during interaction through which the person reaches an unprecedented height with respect to use and experience.

Resonance also stems from the theory of ecological or direct perception, which also engendered the term affordance; a term that Norman introduced to the design community. Gibson (1986) used the term in combination with a radio metaphor to clarify the directness of our perceptual system. A radio station broadcasts information, i.e., radio waves at a particular frequency. The detection of radio waves is based on the principle of resonance. Given that many frequencies (stations) reach a receiver from the antenna, proper tuning of the receiver causes a current in it to resonate in response to one of the incoming signals, and not to others. In case of, for example, visual perception, the radio waves in this metaphor stand for light that is structured (broadcasted) by our environment (the radio station). Our eyes (the antenna) let the signals pass through, and we (the radio) must tune in to the information. For example, if we want to write a message, we are tuned in to information in our environment that affords us to write. Thus when a pencil comes into view, our perceptual system resonates to that information (Michaels and Carello 1981).

However, resonance does not only relate to our perceptual-motor skills. It relates to our cognitive and emotional skills too. We resonate with products because we are people with certain needs, desires and intentions, a social and cultural history and position, etc. Consequently, we do not all resonate to the same products. To elaborate on the writing example, one person might resonate with a cheap disposable pen, another person with the fountain pen he got from his grandpa and another person might resonate with the I-Mac that he bought with his savings (Hummels et al. 2003).

Personality and emotions appear to even be coupled physically. Studies have shown that personality is associated with brain reactivity to emotional stimuli and have identified both common and distinct brain regions where such modulation takes place. Functional imaging studies have examined which brain regions respond to emotional stimuli and by using functional Magnetic Resonance Imaging (fMRI) it has been shown that personality traits moderate such brain activation. More specifically, extraversion was correlated with brain reactivity to positive stimuli in localized brain regions, and neuroticism was correlated with brain reactivity to negative stimuli in localized brain regions (Canli et al. 2001)

\section{Defining Resonance for Design}

Seeing all these different aspects and relations with resonance, it seems necessary to define resonance within the area of product design. Although designers often talk about resonance, it is still unclear what is actually meant by it within a design context. It is probably closely related to that feeling when swinging; being perfectly in sync with a product. But if one wants to design for resonance and even measure resonant interaction, one needs a more precise description. The sunglasses exemplify the important aspects required to obtain resonant interaction that are encapsulated in the following definition:

Resonant interaction is the perfect interplay between a person and a product (or object, another person, ....) which evokes strong positive emotions, unique accompanying behaviour, heightened awareness and cognitive processing. Moreover, these feelings, behaviour and thoughts last after the actual moment of interaction.

The assumption is that resonance occurs due to a fit between the product and the kind of interaction on the one hand, and a person's (latent) concerns, value system, personality, skills, senses and effectivities on the other, all within a given context. Both sides 
strengthen, enhance and even elicit a response from each other. The sunglasses for example fit with my concern of being protected from glaring sunlight and accompanying headaches. But the glasses also heighten my latent soft spot for subtlety, design and uncommon, novel solutions. I like to handle refined products and because the sunglasses are so subtle and light, I use them in a delicate way with gentle gestures. Moreover, I not only like designerly products, but I also like to display them and be associated with them.

What comes to the characteristics of resonance, we can note the following things. Firstly, with the given definition I focus on the positive side of resonance. Secondly, being in sync changes a person's feelings, behaviour, and thoughts. For example, I experience the thrill of taking the sunglasses out of their case and gently putting them on with both hands, which is a perfect subtle feeling. Moreover, I feel more intense and present, and I have the impression that I become taller when wearing them. But resonance also effects my thoughts and cognitive processes. During resonant interaction the product becomes 'present-at-hand' every now and then. This means that the attention is drawn to the product itself instead of to the things you want to do with the product. In the latter case, Heidegger speaks of 'readyto-hand', i.e. the product is absorbed in our everyday dealings with the world around us (Coyne and Snodgrass 1993; Verbeek and Kockelkoren 1998). Resonance evokes positive surprise and awareness during or after use, as the example of the sunglasses also shows. These moments of awareness alternated with the perfect sensation during use let a person move between the three levels of emotional reaction as Norman describes them: visceral (perceptually based), behavioural (expectation based) and reflective (intellectually based) (Norman and Ortony 2006). Usability studies often aim for 'ready-to-hand' and flow, but it appears that 'presence-at-hand' also has its value during interaction.

Thirdly, it is presumed that resonance lasts after the actual moment of interaction, in both the short and long term. It concerns more than just a pleasant or properly functioning product. For example, a shortterm effect can occur when resonating with a gas cooker, which can make one think that the cake is more beautiful when it leaves the oven and that it tastes even better. A long-term effect is coupled to learning to use a product, getting to the bottom of it and becoming an expert, which intensifies the bond and increases the level of resonance. This prolonga- tion is slightly different than the definitions from physics and the ecological perception theory. Within a design context, the metaphor of the swing stands for the total relationship between the product and the person. So, interacting several times with the product can heighten the swing and the level of resonance. When not using the product actively, thinking about it still evokes positive images, memories and emotions. In addition to this time-related aspect, the level of resonance is also affected by a person's concerns, personality, value system, skills, senses, effectivities, the personal history that a person creates with a product over time, and the method of acquisition. This is a dynamic process, thus the level of resonance can change over time.

In order to obtain resonance, the product and interaction must meet a certain standard of performance and behaviour. This does not mean that the product has to be perfect or respect the general principles determined by usability studies, but it should not evoke negative surprise. For example, women can experience resonant interaction with their stiletto heel shoes, despite the fact that they might feel a bit uncomfortable. Wearing these shoes can comply with their standard of performance: the shoes look uncomfortable so it is not surprising that they are a bit uncomfortable when worn. Moreover, these women might experience this discomfort as a natural consequence of, or even add-on to, being beautiful and looking feminine. But shoes that look comfortable, but are uncomfortable to walk in while the owner prefers comfort, will not resonate due to conflicts with the owner's standard of performance. This standard of performance can change over time, which means that resonance can appear on the scene after a while or fade away.

The standard of performance was met by my sunglasses. The sunglasses appeared rather fragile at first sight, but were pretty sturdy when used, which even increased my level of resonance. But there was a downside to the story in the beginning. Having to put the glasses away due to changing lighting conditions conflicted with my rather messy, easygoing personality, in combination with my curly hair, which did not allow me to wear them on top of my head. The new version resolved this problem. The special polycarbonate polarized lenses that offer $100 \%$ UV protection enable me to see perfectly indoors. 


\section{Researching Resonance through Design}

Since resonance is extremely personal, context- dependent and experience-related, studying and measuring this phenomenon calls for a research method that can take these issues into consideration. Research through design, a concept first coined by Archer when talking about 'research through practice' (Archer 1995), seems a useful approach that can address this kind of complexity. By designing in a way that results in experiential prototypes, and subsequently testing these prototypes, scientific knowledge can be generated about resonant interaction.

The strength and beauty of research through design lies in its ability to address the complexity of all interlinked aspects such as users, context, design and technology. It can address the user's experience, which is hard with traditional approaches. Therefore, it is more an approach that searches for conditional laws instead of general laws. Finally, research through design is also a method that generates physical hypotheses and not just a way of testing hypotheses. A designer-researcher places objects in the world, and because the studied phenomena are extremely complex, he usually gets unforeseen reactions and employs the user as a sparring-partner to generate hypotheses.

My studies on resonant interaction have two main goals: verifying the postulated definition of resonance as shown and explained above, and finding salient aspects of resonant interaction that can help designers to tune their products towards resonant interaction. The latter can enhance diversity at an interaction and experiential level, which I consider to be of paramount importance when pursuing the next generation of digital products. The results of the first studies encouraged me to study the influence of four salient modes on resonance (Hummels and van der Helm 2004):

- Interaction mode: What style of interaction is used? What is the influence of 1) physical aspects of the interaction such as interactivity (e.g. complexity, type of bodily movements, action-feedback mapping) and vividness (e.g. richness and abundance), 2) experiential aspects such as engagement, flow, and excitement, and 3) a combination of both e.g. expressivity (product and behaviour) (Frens 2006; Wensveen et al. 2004)?

- Activity mode: What is the character of the interaction? For example, is the user aiming for an experience-oriented activity or a goal-oriented activity (Hassenzahl et al. 2002), and does the type of interaction enhance usability and straightforward use and/or experience and exploration?

- Human mode: Who is interacting with the product (characteristics, belief, values, concerns, personality, skills, senses, effectivities ...) and what is the social dimension, e.g., are people working together or alone?

- Context mode: Where, when, and how often, how long, how frequently, etc. is a product used?

In order to study these four modes and the two main goals (verifying the definition and finding salient aspects), I am gathering people's beliefs, dreams and product examples that evoke resonance and I am designing, building and testing a variety of products and installations. In this way I am trying to obtain information about why resonance occurs, and what the characteristics and conditions are that allow it to occur. Moreover, it allows me to find patterns and formulate guiding principles for design that can awaken and amplify resonant interaction. Due to the complexity of the phenomenon, every study has its own focus and the combination is supposed to create the overall framework.

In this paper I discuss two studies in particular 'Design for personality' and 'Prolongation of positive emotions'. These projects illustrate the way of working and the kind of information I am trying obtain.

\section{Designing Resonance for Personality}

Have you ever thought about gently caressing your music player to get it to play relaxing music? Probably not if you look at the present range of MP3 players which are operated by buttons, (touch-sensitive) scroll wheels and sliders. Designed by Master student Johannes Zachrisson, Puppy is one of the designs made for the course Interactive Technology Design of the Faculty Industrial Design Engineering, Delft University of Technology (Fig. 1). 21 students explored whether it is possible to design expressive tangible stress-relieving products, specifically music players, for different personalities. Through their designs, they explored the link between the interaction mode and the human mode. Puppy is different and likes to be caressed, unlike existing MP3 players. This soft furry MP3 


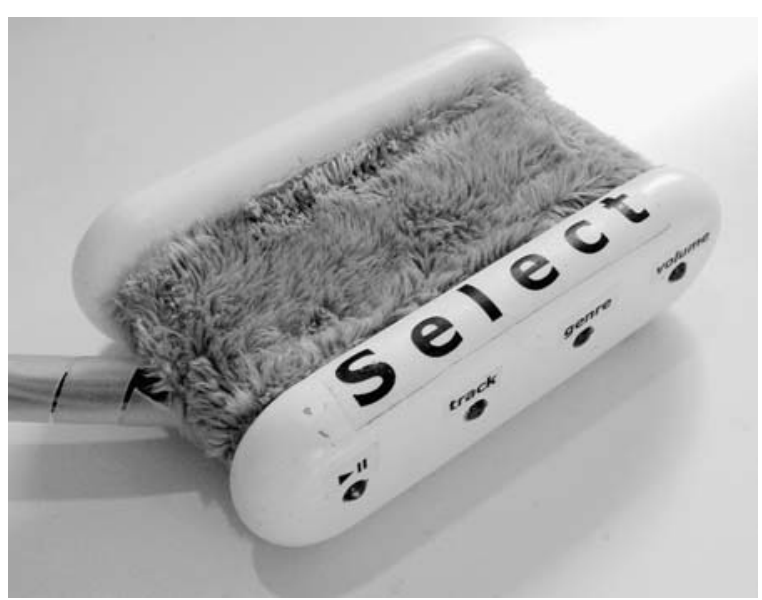

Fig. 1 Stroking on the select side of Puppy allows one to switch between functions (play/pause, track, genre, volume) and stroking on the opposite $+/-$ side increases or decreases the chosen function. The direction of stroking and the character of the stroke (speed, area of touching and the length of the stroke) determine how the function is interpreted and executed

player, which is designed to reduce stress, is operated by stroking it, where place, direction and character of the stroke determine how the functionality of the player is interpreted and executed.

\section{Myers Briggs Type Indicator and Human Value Theory}

The personalities that the students focused on were defined using the Myers Briggs Type Indicator (MBTI) (Briggs 1987) and the Human Value Theory from Schwartz (Schwartz 1992). MBTI is based on the theory that every individual has a primary mode of operation within four categories. Each personality type consists of four letters corresponding to one of the modes within a category:

- Introversion (I) vs. Extraversion (E). Our flow of energy defines how a person receives the essential part of his or her stimulation, from within (I) or from external sources $(\mathrm{E})$.

- Sensing (S) vs. Intuition (I). Does a person take in information using the five senses (S) or does he or she rely on instincts (I)?

- Feeling (F) vs. Thinking (T). How does a person prefer to make decisions, based on personal subjective value systems (F) or based on logic and objective considerations (T)?

- Judging (J) vs. Perceiving (P). How does a person deal with the daily world, in an organised and structured way $(\mathrm{J})$ or is he or she flexible and likes alternation $(\mathrm{P})$ ?

Next to MBTI, we also used the Human Value Theory from Social psychologist Shalom H. Schwartz. He provides a definition of human values describing five formal features the concept incorporates: 'Values are (1) concepts or beliefs, (2) pertain to desirable endstates or behaviours, (3) transcend specific situations, (4) guide selection or evaluation of behaviour and events, and (5) are ordered by relative importance.' (Schwartz 1992). Schwartz identified and evaluated a comprehensive set of 56 values, including concepts like Social Power, Creativity and Pleasure, and developed a universal structure that organises values in a two-dimensional plane. This structure of human values reveals how compatible or conflicting values are. For example, more conflict exists between Social Power and Helpful than between the more compatible values Humble and Helpful. Schwartz grouped the set of values into 10 motivationally distinct value types (Ross et al. 2007) Fig. 2 shows the graphical representation of the theoretical structure of human values.

\section{The Design Process}

Every team got a different MBTI type and a different set of values. For example, Puppy was designed for an Introvert Sensing Feeling Judging (ISFJ) type, i.e., a person that obtains stimulation from within (I), takes in information using all senses (S), makes decisions based on a personal subjective value system (F) and that deals with the world in an organised and structured way $(\mathrm{J})$. Moreover, Puppy is designed for someone who considers benevolence, security and tradition to be important personal values.

The students went through several design loops, each with a specific focus such as expressivity and adaptability, to produce and test their final prototype. These different aspects were explored through interactive tangible sketches, i.e., 3D sketchy models, generally with sensors, which can be used and tested at an experiential level. Key aspects of this design technique are the focus on rich tangible interaction, the speed of making and testing a 3D tangible sketch, and the experiential and interactive character of the sketch. We incorporated Phidget Interfacekits, a user interface building kit including an extensive set of (plug-and-play) sensors 
Fig. 2 The structure of 56 human values, including the demarcation lines of the 10 value types (Schwartz 1992)

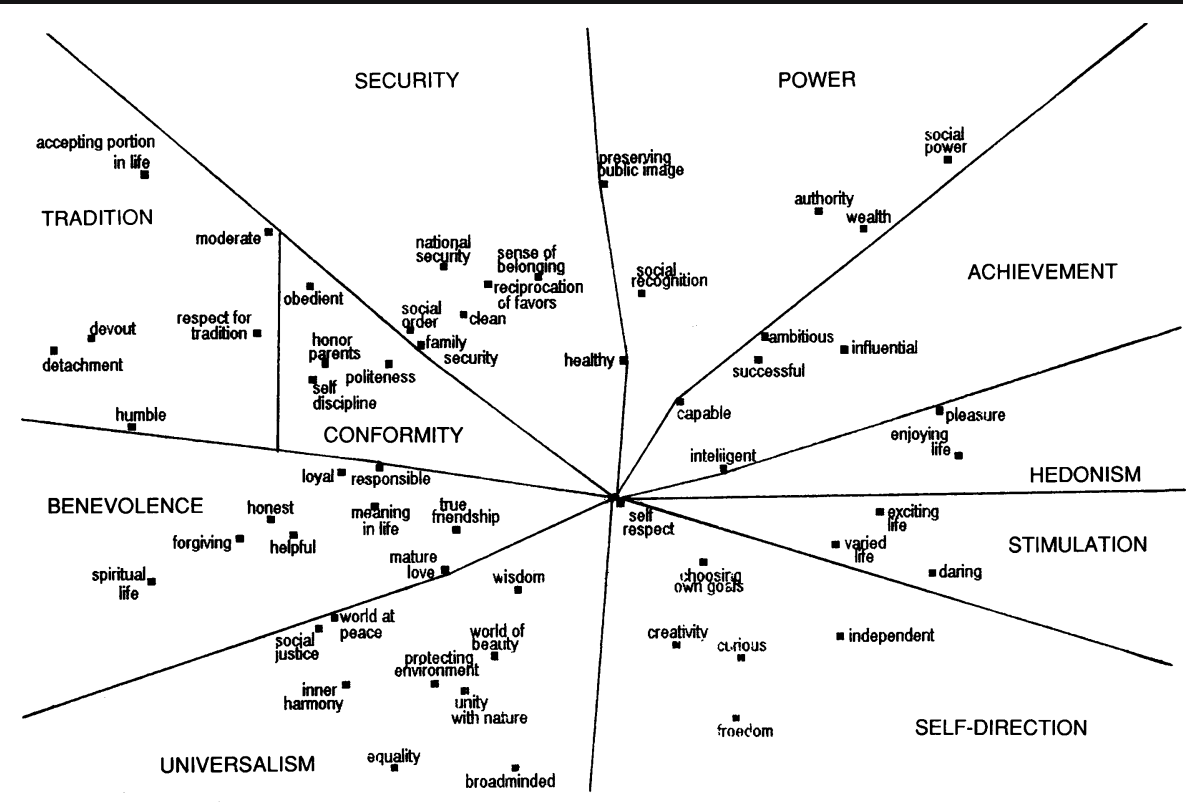

and actuators, in the models. The kits were connected to a computer using a software development environment based on real-time dataflow modelling. This approach allows designers to iterate quickly from idea to prototype, because they can change the program while it is running (Hummels et al. 2006).

After every team had created a persona based on a specific MBTI type and set of human values, they designed and built their first interactive tangible sketch of a music player with basic functionality specifically for their persona. Next they focused on the expressive quality of the interaction. What kind of interaction would suit their persona? After creating a so-called 'choreography of interaction' (Klooster and Overbeeke 2005), an expressive music player was designed and built based on the dynamic quality of the movements, the physical involvement in these movements and the expressed meaning of these movements. Subsequently the third interactive tangible sketch was built aiming at detecting the level of stress and changing the music and the functionality based upon this information. Finally, every team built their final high-fidelity prototype based on the previous findings.

Testing the Designs

In order to test if it is possible to design expressive tangible stress-relieving products for different personalities, a user test was conducted to determine whether users preferred the prototypes designed for a personality type that matched their own personality type. In total, 11 designs were tested by 44 participants of whom half of them had a background in design and the other half were laymen in design.

After seeing a 1.5 minute film clip explaining the design, the participants assessed the product including the interaction characteristics using the following criteria:

- Keywords. The participant described the design using three keywords.

- Schwartz's human value map. The participant selected maximally three values or value types that he considered to be evoked by the designs.

- MBTI classification. The participants judged the design with respect to the four MBTI categories (E/I, N/S, T/F and J/P) using a 7-point scale.

- Hassenzahl's AttrakDiff test (Hassenzahl et al. 2003). The participant evaluated the design using 29 7-point scales with pairs of words that represent extreme contrasts with respect to pragmatic and hedonic qualities of the design.

- Top three players. The participants selected the top three players that they preferred as a whole, and the top three that they considered reduced their stress the best.

After this assessment, all participants were able to try out the prototypes and adjust their assessment if required. 
Table 1 Summary of interaction patterns

\begin{tabular}{ll}
\hline Interaction & Description \\
\hline $\begin{array}{l}\text { Pleasant exciting interaction } \\
\text { Unfamiliarity }\end{array}$ & The property of being free from conventional and routine procedure \\
Learnability & The exploration required to learn the methodology \\
Coincidence & The unexpected consequence not to be planned or arranged \\
Continuity & The behavioural property of being free from discreet and linear actions \\
Pleasant clear interaction & \\
Acceptability & The property of being well-accepted by the majority of people \\
Intuitiveness & The property of being easily understandable \\
Consistency & The property of being free from logical inconsistencies \\
Simplicity & The property of being free from complexity and confusion \\
Pleasant exciting-clear interaction & \\
Generally implies the patterns of both pleasant-exciting-interaction and pleasant-clear-interaction plus: \\
Visuality & The rich and active feedback through visual media \\
Modularity & The functional independence by employment of physical or virtual modules \\
Customisability & The possibility to customise the interface \\
\hline
\end{tabular}

Results and Discussion

The results suggested that it is possible to design for personality. For example, the participants labelled Puppy as a benevolence- and security-evoking player which tallies with the starting-points for this design, although it is less traditional than expected. Moreover, according to the participants, the player is considered to fit a $\operatorname{ISF}(\mathrm{P} / \mathrm{J})$ type, which is correct apart from the last aspect: the way that the person deals with the world. Puppy is designed for people who are organised and structured $(\mathrm{J})$, but the design doesn't have a clear preference for either judging $(\mathrm{J})$ or perceiving $(\mathrm{P})$.

However, the results also show that not all designers are able to design for personality; yet! These results are in accordance with our previous studies that show the same pattern (van der Heijden et al. 2005). The results of this test and the results of the preceding ITD course with a similar project will be further analysed to gain insight into the salient aspects of design for personality, in order to support more designers to create personalised designs. Moreover, the appropriateness and validity of MBTI and value structures to obtain personalised designs will be further studied.

\section{Prolongation of Positive Emotions}

The second study focuses on general interaction characteristics that could evoke resonance. As indicated in its definition, it is presumed that resonance lasts after the actual moment of interaction, both short term and long term. It concerns more than just a pleasant or properly functioning product. Han Chul Jung tested this presumption in his final project before obtaining his Master's degree Design for Interaction at the Faculty of Industrial Design Engineering, Delft University of Technology (Jung 2005).

This graduation project tried to find salient aspects during tangible interaction (interaction mode described in Researching Resonance through Design Section) that cause prolongation of positive emotions.

Finding Interaction Factors that Evoke Positive Emotions

In order to answer the question 'Which interaction factors evoke positive emotions during tangible interaction?' Han Chul Jung studied a large set of design concepts of music players that were designed for MBTI personality types and tested during two user studies within the ITD course, comparable to the project described in the previous chapter. During the first of these user tests, eight teams tested their 16 designs concepts, which were presented on paper (one design per MBTI type), with on average 20 participants (154 participants in total). All these concepts were evaluated by using 14 bi-polar 10-point scales that were related to the interaction qualities of the design and the personal impression of the participant.

For the analysis phase, Jung included 93 of the 128 design concepts. Correlations, factor analyses and 
Fig. 3 EmoCards plus 9point scales, including the data transformation to obtain an ordinal order per cell, e.g. the cell with the red spot scores 5.7 points

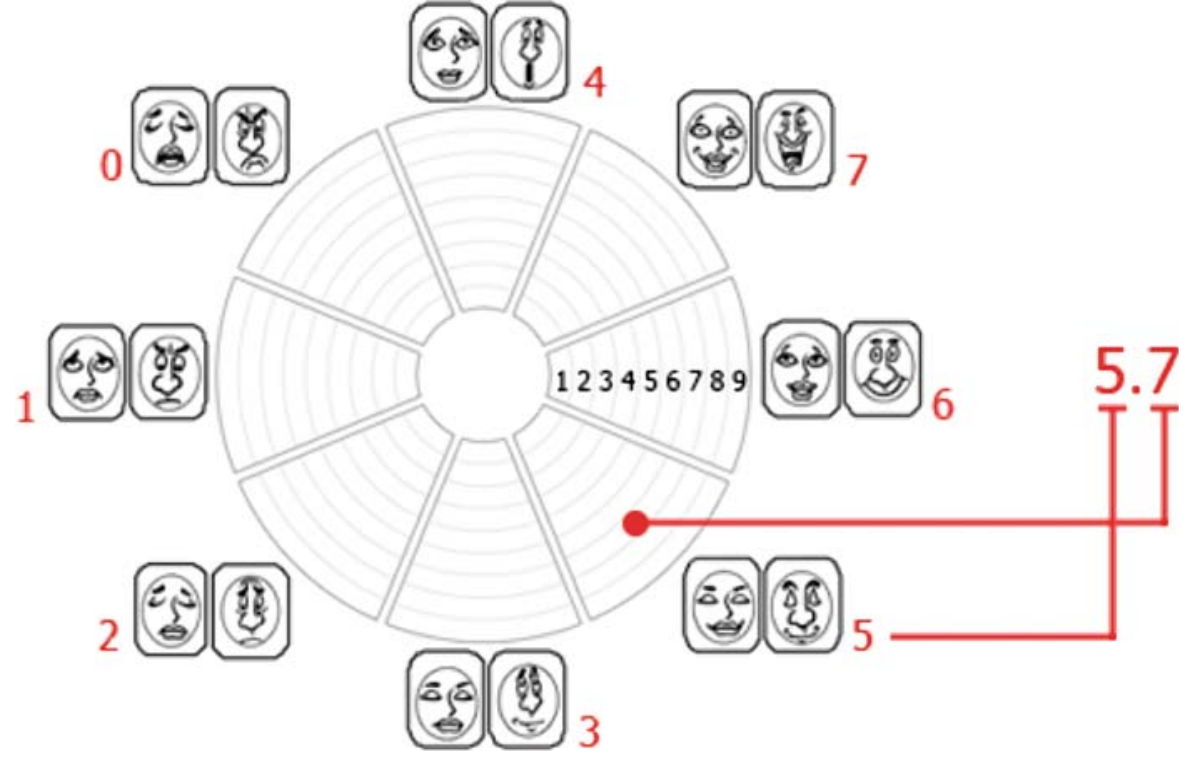

regression analyses were performed to find the interaction factors that evoke positive emotions during tangible interaction, i.e., a mix of pleasant emotions ranging from low to high arousal, although the later is preferred. He found that both exciting interaction and clear interaction can evoke pleasure during physical interaction. That is, on the one hand, richness, variety, excitement and challenge influence positive emotions. On the other hand, this is also the case for clearness, ease of use, and controllability. This seems to concur with studies carried out by Hassenzahl (2004) and Norman (2002).

But what kind of product and interaction features make interaction exciting, challenging, and rich, or clear, easy, and controllable? This question was answered by clustering the designs that score high on pleasantness as well as on exciting interaction, or clear interaction or exciting-clear interaction, and those designs that score low on one or both of these aspects. Subsequently, the similarities and differences between these designs with respect to interaction characteristics were labelled. This resulted in patterns for the three types of interaction, as shown in Table 1.

\section{Music Players that Prolong Positive Emotions}

It was hypothesised that after the physical interaction with a product stops, the combination of exciting and clear interaction can prolong positive emotions more than exciting interaction or clear interaction would.
To test this hypothesis three music players were designed, one for every condition. The first prototype aimed for clear interaction, the second one to enhance excitement and the third prototype combined both aspects, based on the pattern described in Table 1 . The expectation was that all three prototypes would initially evoke the same amount of positive feelings, but that the last prototype would achieve the highest level of prolongation.

All music players consist of an interactive board with a projected interactive animation and a set of tangible objects. Each of the players has a different set of three tangible objects to 1) select an artist 2) select a song and 3) control the volume.

\section{Testing the Prototypes}

Twenty-eight design students tested one of the three prototypes several times, using a between-subjects approach. In this set-up the between-subjects factor was the type of interaction, i.e., every subject evaluated one of the three designs, which had either exciting, clear or exciting-clear interaction. The within-subjects factor was time, i.e., the music players were assessed four times in two days.

The experiment started in the ID-StudioLab where the participants were invited to try out and play with the assigned music player for \pm 3 minutes. They assessed the player immediately after using it. The subsequent three assessments were conducted at home, separated by 


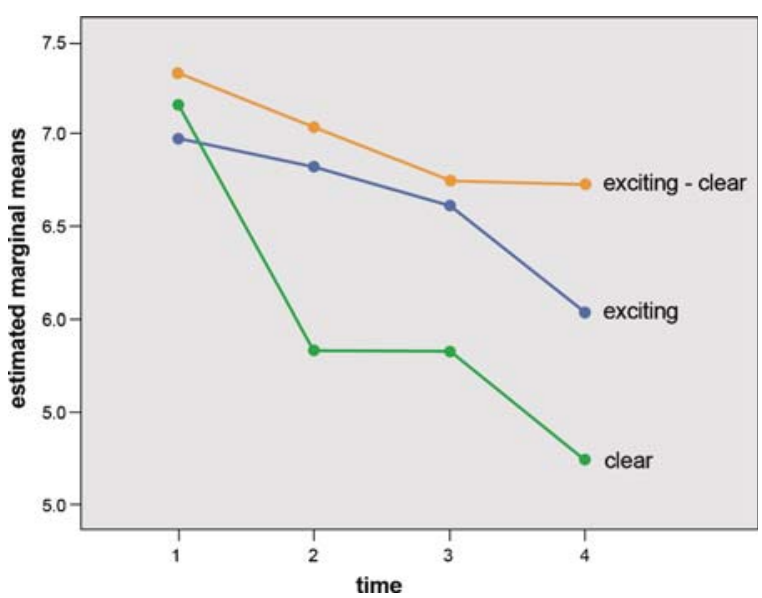

Fig. 4 The mean pleasant feeling (vertical axis) per condition was calculated for every assessment period (horizontal axis), showing a quick decline for 'clear interaction', a first slow but at last quick decline for 'exciting interaction, whereas the pleasant feeling for 'exciting-clear' only decreases very slowly

12-hour intervals. The within-subjects variables that were observed were emotional status, eager-to-reuse, and the products' excitement and clearness. The first variable was measured using Emocards (Desmet et al. 2001) that were extended with 9-point scales, see Fig. 3. The subjects were asked to select a facial expression that best reflected their feelings towards the music player at that moment. The faces were distributed around a circle using 'pleasantness' on the horizontal axis and 'arousal' on the vertical axis. The other variables were measured using 9-point bi-polar scales. In this paper we will only address the emotional status variable.

\section{Results and Conclusion}

Immediately after the first test, all music players generally evoked similar positive emotions $(92 \%$ of the participants felt pleasure), which complies with the short-term pleasure component of resonant interaction. However, after the fourth and last assessment, the participants who tested the exciting product felt Mid-Arousal or Low-Arousal Pleasant, the participants for the clear product felt Low-Arousal Pleasant and even Low-Arousal Neutral. However, the participants who had played with the exciting-clear product still felt High-Arousal and Mid-Arousal Pleasant. Using a mixed between-within subjects ANOVA, the main effect within subjects, the interaction effect within subjects and the between subject effect were statistically significant $(p=0.04)$. (Fig. 4).
So we can conclude that after physical interaction with a product stops, the combination of exciting and clear interaction can prolong positive emotions more than exciting interaction or clear interaction would, which confirms the last part of the definition of resonant interaction.

\section{Overall Conclusions}

People are all different, so why are most digital products alike, particularly at an interaction level? Resonance could be a concept to stimulate designers to address the diversity of people, because resonant interaction is the perfect and unique interplay between a person and a product (or object, another person, ....,) It evokes strong positive emotions, unique accompanying behaviour, heightened awareness and cognitive processing and it is presumed that these feelings, behaviour and thoughts last after the actual moment of interaction.

Research through design appears to be an approach to study resonant interaction and find its salient aspects. The designs and studies show not only that it is possible to enhance pleasantness during and after interaction, but also that one can formulate salient aspects that evoke this prolongation of pleasure, thus taking the first step towards designing for resonant interaction.

Moreover, it appears that it is possible, at least for some designers, to design for personality and address personal preference for a certain type of interaction, including the social values it evokes.

Although this knowledge about diversifying design could be applied in many ways, I personally hope that it also increases awareness of common values such as freedom, dignity and brotherhood, as expressed in the Universal Declaration of Human Rights. Designing for resonant interaction and diversity gives us the opportunity to respect the individual, hopefully without forgetting that we can be individuals only in a social context, by respecting each other and taking responsibility for society.

Acknowledgements I would like to thank Han Chul Jung, Johannes Zachrisson, Kees Overbeeke, Aadjan van der Helm, Rob Luxen, Paul Hekkert, David Keyson, Philip Ross, as well as all participating ITD students and subjects, without whom this search for resonant interaction would have turned into a destitute journey, instead of this inspiring and sparkling one. 


\section{References}

Archer, B. (1995), The nature of research. Co-Design Journal (2): 11 .

Briggs, K. C. (1987), Myers-Briggs Type Indicator. Mountain View, CA: Consulting Psychologists Press.

Canli, T., Zhao, Z., Kang, E., Gross, J., Desmond, J. and Gabrieli, J. (2001), An fMRI study of personality influences on brain reactivity to emotional stimuli. Behavioral Neuroscience 115 (1): 33-42.

Coyne, R. and Snodgrass, A. (1993), Rescuing CAD from Rationalism. Design Studies 14 (2): 100-123.

Desmet, P. M. A., Overbeeke, C. J., and Tax, S. J. E. T. (2001), Designing products with added emotional value: development and application of an approach for research through design. The Design Journal 4(1): 32-47.

Dourish, P. (2001), Where the action is: The foundation of embodied interaction. Cambridge: MIT Press.

Frens, J. W. (2006), A rich user interface for a digital camera. Personal and Ubiquitous Computing 10(2-3): 177-180.

Gibson, J. J. (1986), The Ecological Approach to Visual Perception. Hillsdale NJ: Lawrence Erlbaum Associates, Inc. (original work published 1979)

Hassenzahl, M. (2004), Emotions can be quite ephemeral. We cannot design them. Interaction Magazine 11(5) : 46-48.

Hassenzahl, M., Burmester, M. and Koller, F. (2003), AttrakDiff: ein fragebogen zur messung wahrgenommener hedonischer und pragmatischer qualität (german). In Mensch \& Computer 2003. Interaktion in Bewegung, edited by J. Ziegler and G. Szwillus, 187-196. Stuttgart, Leipzig: B.G. Teubner.

Hassenzahl M., Seifert, K. and Pastoor, S. (2002), The effect of "usage modes" on product appeal., http://www.tudarmstadt.de/fb/fb3/psy/soz/forvero_mh.html.

Hummels, C., Overbeeke, C. J. and Klooster, S. (2006), Move to get moved: a search for methods, tools and knowledge to design for expressive and rich movement-based interaction for design. Personal and Ubiquitous Computing [online]. http://dx.doi.org/10.1007/s00779-006-0135-y, accessed November 2006.

Hummels, C., Ross, P. and Overbeeke, C. (2003), In search of resonant human computer interaction: building and testing aesthetic installations. In Proceedings of the 9th International Conference on Human- Computer Interaction (Interact), Zurich, Switzerland, September 2003, edited by M. Rauterberg, M. Menozzi and J. Wesson, 309-406. Amsterdam: IOS Press.

Hummels, C. C. M. and van der Helm, A. (2004), ISH and the search for resonant tangible interaction. Personal and Ubiquitous Computing 8(5): 385-388.
Jung, H. C. (2005), Resonant tangible interaction + prolongation of positive emotions. Delft: Unpublished Master thesis, Delft University of Technology.

Klooster, S. and Overbeeke, C. J. (2005), Designing products as an integral part of choreography of interaction: The product's form as an integral part of movement. In Design and Semantics of Form and Movement, edited by L. Feijs, S. Kyffin and B. Young, 23-35. Eindhoven: Koninklijke Philips Electronics N.V.

Michaels, C. F. and Carello, C. (1981), Direct perception. Englewood Cliffs: Prentice-Hall.

Norman, D. A. (2002), Emotion and Design: Attractive Things Work Better. Interaction Magazine 9(4): 36-42.

Norman, D. A. and Ortony, A. (2006), Designers and Users: Two Perspectives on Emotion and Design. In Theories and Practice in Interaction design, edited by S. Bagnara, and G. Crampton-Smith, 91-103. Mahwah, N.J.: Lawrence Erlbaum associates.

Overbeeke, C. J., Djajadiningrat, T., Hummels, C., Wensveen, S. and Frens, J. (2003), Funology: From Usability to Enjoyment. In Let's Make Things Engaging, edited by M. A. Blythe, A. F. Monk, C. J. Overbeeke and P. C. Wright, 717. Dordrecht: Kluwer.

Ross, P., Overbeeke, C. J., Wensveen, S. and Hummels, C. (2007), A designerly critique on enchantment. Personal and Ubiquitous Computing [online]. http://www.springerlink. com/content/4746711475462200/fulltext.pdf, accessed June 2007.

Schwartz, S. H. (1992), Universals in the content and structure of values: theoretical advances and empirical tests in 20 countries. In Advances in Experimental Social Psychology, edited by M. P. Zanna, 25: 1-65. San Diego, CA: Academic Press.

Smith, D. (1974), A case study and analysis of the Tacoma Narrows Bridge failure. 99.497 Engineering Project. Ottawa, Canada: Department of Mechanical Engineering. Carleton University.

van der Heijden, A., Hennink, A, Kistemaker, N. and Hummels, C. (2005), Especially made for you. In Proceedings of the conference Designing Pleasurable Products and Interfaces, Eindhoven, 24-27 October, the Netherlands, edited by S. Wensveen, E. Diederiks, T. Djajadiningrat, A. Guenand, S. Klooster, M. Stienstra, P. Vink and K. Overbeeke, 478-479. Eindhoven: TU Eindhoven.

Verbeek, P.-P. and Kockelkoren, P. (1998), The things that matter. Design Issues 14(3): 28-42.

Wensveen, S. A. G., Djajadiningrat, J. P., and Overbeeke, C. J. (2004), Interaction Frogger: a design framework to couple action and function. In Proceedings of the 2004 Conference on Designing Interactive Systems: Processes, Practices, Methods, and Techniques, August, 2004, 177-184. Cambridge, MA, USA. 